\title{
Temperature dependence measurements of the supercurrent-phase relationship in niobium nanobridges
}

\author{
A. G. P. Troeman, ${ }^{1}$ S. H. W. van der Ploeg, ${ }^{2}$ E. Il'Ichev, ${ }^{2}$ H.-G. Meyer, ${ }^{2}$ A. A. Golubov, ${ }^{1}$ M. Yu. Kupriyanov, ${ }^{3}$ and \\ H. Hilgenkamp ${ }^{1}$ \\ ${ }^{1}$ Faculty of Science and Technology and Mesa ${ }^{+}$Institute for Nanotechnology, University of Twente, P.O. Box 217, 7500 AE Enschede, \\ The Netherlands \\ ${ }^{2}$ Department of Cryoelectronics, Institute for Photonic Technology, D-07702 Jena, Germany \\ ${ }^{3}$ Nuclear Physics Institute, Moscow State University, 119992 Moscow, Russia
}

(Received 16 August 2007; published 14 January 2008)

\begin{abstract}
The current-phase relationship has been measured as a function of temperature for niobium nanobridges with different widths. A deformation from Josephson-like sinusoidal characteristics at high temperatures to sawtooth shaped curves at intermediate and multivalued relationships at low temperatures was observed. Based on this, possible hysteresis in the current-voltage characteristics of niobium nanobridge superconducting quantum interference devices can be attributed to phase slippage.
\end{abstract}

DOI: 10.1103/PhysRevB.77.024509 PACS number(s): 74.78.Na, 74.45.+c, 74.50.+r, 85.25.Cp

\section{INTRODUCTION}

One of the fundamental characteristics of superconducting structures is the relationship between the supercurrent through and the phase difference across the structure. The prediction $^{1}$ and first experimental verification ${ }^{2}$ of periodical current-phase relationships (CPRs) in superconductorinsulator-superconductor tunnel junctions triggered the onset of research on Josephson devices. Much of the classical studies on this subject focused on structures where two superconducting layers are separated by a barrier with a thickness of the order of the superconducting coherence length $(\xi)$. With the advances in nanotechnology, the development of superconducting structures with lateral dimensions of the order of $\xi$ also became possible. Examples of such systems are superconducting nanobridges, which, when of sufficiently small dimensions, are known to exhibit a periodical CPR. ${ }^{3,4}$ Based on this similarity to classical Josephson tunnel junctions, the development of superconducting quantum interference devices (SQUIDs) incorporating two of such nanobridges has been a topic of ongoing research, e.g., for the detection of the magnetization reversal of small magnetic clusters ${ }^{5-7}$ and in scanning SQUID microscopes. ${ }^{8-11}$ The application of superconducting nanobridges as single photon detectors, for instance, as described in Ref. 12, has been a topic of interest in recent years. In addition, hot electron bolometers based on superconducting nanobridges are explored as detectors in astrophysical observations at terahertz frequencies. ${ }^{13}$ Recent fundamental interest in superconducting nanobridges has furthermore been motivated by the possible application of such structures in flux qubits. ${ }^{14,15}$

Even though applications based on the Josephson-like characteristics of superconducting nanobridges have been investigated extensively, up until now, the exact nature of the CPRs in these structures has mainly been studied theoretically. It is predicted to be dependent on the dimensions of the structure. According to the Kulik-Omelyanchuk model, ${ }^{16}$ a gradual temperature $(T)$ dependent deformation of the CPR from sinusoidal at high $T$ to sawtoothlike at lower $T$ is expected in the clean limit. Strongly nonsinusoidal CPRs at low $T$ are also predicted in the diffusive regime for nanowires with lengths $L \ll\left(\xi_{0} l\right)^{1 / 2}$ (where $\xi_{0}$ is the BCS coherence length and $l$ the electronic mean free path) and small transverse sizes $W \ll L$. These models were qualitatively verified for the CPR in clean ballistic niobium point contacts. ${ }^{17}$ Recent quantitative agreement between experiment and theory was reported for aluminum atomic contacts. ${ }^{18}$ Likharev and Yakobson first considered the effect of an increasing weak link length on the CPR for structures in which the temperature is close to the critical temperatures of both the electrodes $\left(T_{c}\right)$ and the nanobridge $\left(T_{c}^{\prime}\right) .{ }^{19}$ Their model describes a similar deformation of the CPR, from sinusoidal to sawtoothlike, as a function of increasing bridge length. Furthermore, at a critical length $L \gtrsim 3.5 \xi_{0}(T)$, with $\xi_{0}(T)$ the Ginzburg-Landau (GL) coherence length, the nature of the CPR becomes multivalued. In this limit, superconductivity is suppressed above the critical current by phase slippage of the superconducting order parameter $(\Psi)$ in the structure. During a phase slip, the order parameter fluctuates to zero, allowing the relative phase to relax by $2 \pi$ and resulting in a voltage pulse. In the GL regime, this model was extended to two dimensions in Ref. 20. For wide $[a \gtrsim 3.5 \xi(T)]$ and long $[L \gtrsim 4.4 \xi(T)]$ nanobridges, the coherent motion of vortices across the structure is expected to determine the CPR.

For fixed values of the bridge length and $T_{c}=T_{c}^{\prime}$, the described deformation of the CPR and crossover as a function of decreasing temperature were discussed quantitatively by Kupriyanov et $a .^{21}$ within a model valid at arbitrary temperatures. In terms of the GL approach, this can be explained by the fact that $\xi(T)$ increases as a function of temperature, which, for fixed $L$, is physically similar to a decrease of the effective bridge length. Based on the models discussed above, the predicted transition of the CPR in a superconducting nanobridge $\left[L \gtrsim\left(\xi_{0} l\right)^{1 / 2}\right]$ is qualitatively depicted in Fig. 1. The dotted line corresponds to the sharp drop in phase related to the phase slip mechanism.

Previously, the described crossover was studied only indirectly in experiment by measuring the power dependence of Shapiro steps in the current-voltage $(I V)$ curves of microwave-irradiated Sn microbridges. ${ }^{22}$ This study con- 


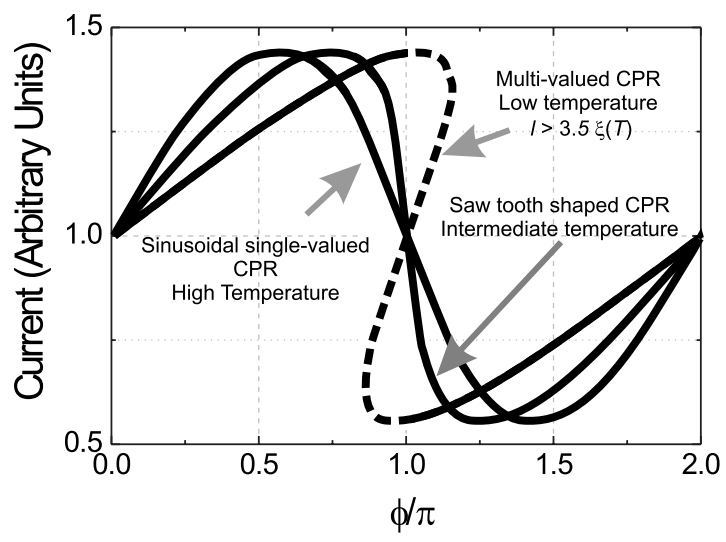

FIG. 1. Schematical representation of the temperature dependent deformation of the CPR of a superconducting nanobridge. The dotted part of the multivalued characteristic corresponds to the nucleation of a phase slip center in the structure.

firmed the existence of a boundary between the Josephson effect and the coherent motion of Abrikosov vortices in the bridges. Also, direct experimental observations of the deformation of the CPR in the single-valued regime for indium microbridges were reported in $1980 .{ }^{23}$ The transition to the regime where phase slippage of the superconducting order parameter determines the CPR was, however, not verified in this study.

In this paper, we describe direct measurements of the temperature dependent $\mathrm{CPR}$ on niobium nanobridges, patterned by means of focused ion beam (FIB) milling. We demonstrate the cross-over from the Josephson effect to multivalued characteristics corresponding to the nucleation of phase slip centers in the structure due to current induced depairing effects. The results agree qualitatively with theoretical predictions.

\section{EXPERIMENT}

The conducted CPR measurements were based on a method where the weak link of interest is incorporated in a superconducting loop of small inductance $\left(L_{L}\right){ }^{24,25}$ This ring is inductively coupled to a tank circuit with inductance $L_{T}$ and high quality factor $(Q)$ via a mutual inductance $(M)$. The tank circuit is driven by a dc bias $\left(I_{d c}\right)$ current and a superimposed radio frequency current $\left(I_{r f}\right)$ with a frequency $\omega_{0}$ close to its resonance frequency. The measurements are based on monitoring the phase shift $\alpha$ between the tank circuit voltage $U$ and $I_{r f}$. For sufficiently small signals, this results in the following relationship: ${ }^{26}$

$$
\tan \alpha=\frac{M^{2} Q}{L_{T} L_{L}} \frac{\beta f^{\prime}(\phi)}{1+\beta f^{\prime}(\phi)},
$$

with $\beta=2 \pi L_{L} I_{c} / \Phi_{0}$ and $I_{s}(\phi)=I_{c} f(\phi)$. In this equation, $I_{c}$ is the critical current of the weak link and $I_{s}$ the supercurrent passing through it, with $\Phi_{0}$ being the magnetic flux quantum $\left(h / 2 e \approx 2.07 \times 10^{-15} \mathrm{~Wb}\right)$. The phase difference $\phi$ across the structure is biased by the external magnetic flux generated by

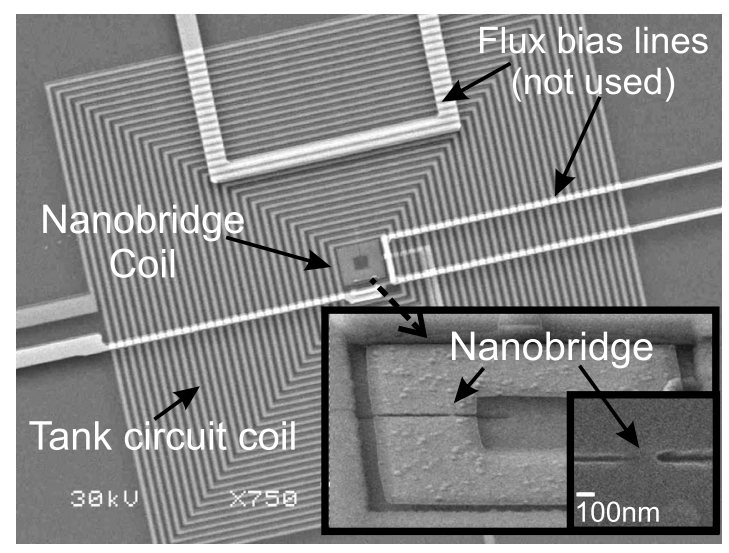

FIG. 2. Scanning electron micrograph displaying the sample layout for CPR measurements. The nanobridge is patterned by means of FIB in a small $\mathrm{Nb}$ ring centered in the tank circuit coil.

$I_{d c}$. The CPR is given by $\beta f(\phi)$ and, if single valued, can be reconstructed from measured $\alpha\left(I_{d c}\right)$ curves.

A scanning electron micrograph showing the geometry of a realized sample is displayed in Fig. 2. All samples are composed of a large $\mathrm{Nb}$ tank circuit input coil (30 turns, $L_{T} \approx 65 \mathrm{nH}$, layer thickness $\approx 200 \mathrm{~nm}$ ) and a small Nb nanobridge coil of $\approx 50 \mathrm{~nm}$ thickness $\left(L_{L} \approx 5.4 \mathrm{pH}\right.$ or $L_{L} \approx 8.3$ $\mathrm{pH}$, depending on the specific geometry) in which the nanobridge is patterned, located in the center of this coil. The additional flux bias lines are not used in the described experiments. Apart from the FIB patterning of the nanobridges, all structures were defined by means of optical and electron beam lithography. The $\mathrm{Nb}$ films and interlaying insulating layers of $\mathrm{SiO}_{x}$ were grown by magnetron sputtering. The actual patterning of the nanobridges is based on a $25 \mathrm{keV} \mathrm{Ga}$ FIB process and is described elsewhere. ${ }^{11}$

\section{RESULTS AND DISCUSSION}

In Fig. 3, several $\alpha\left(I_{d c}\right)$ characteristics recorded at different temperatures are displayed for a sample consisting of a $60 \mathrm{~nm}$ wide, $150 \mathrm{~nm}$ long bridge, patterned in a coil with $L_{L} \approx 5.4 \mathrm{pH}$. To improve the clarity, vertical offsets have been added to the different characteristics. For the curves displayed in the upper part of this graph $(4.18 \mathrm{~K} \leqslant T \leqslant 4.81 \mathrm{~K})$, jumps in the $\alpha\left(I_{d c}\right)$ characteristics [discontinuities in $\alpha^{\prime}\left(I_{d c}\right)$ ] can be discerned. Since $\alpha\left(I_{d c}\right)$ is directly related to $\beta f^{\prime}(\phi)$ through Eq. (1), such dependencies can be explained by discontinuities in the measured CPR of the sample. Qualitatively, this corresponds to a multivalued character of the CPR, as was shown in Fig. 1. It can thus be concluded that at these temperatures, the nature of the CPR is determined by phase slippage in the structure.

For higher temperatures $(T \geqslant 4.98 \mathrm{~K})$, continuous $\alpha^{\prime}\left(I_{d c}\right)$ curves are measured, corresponding to single-valued CPRs (and thus Josephson-like weak link characteristics). From these characteristics, the $\beta f(\phi)$ curves can be reconstructed. The results of such calculations for the $\alpha\left(I_{d c}\right)$ curves displayed in Fig. 3 are shown in Fig. 4. Qualitatively, a deformation from sinusoidal characteristics at high temperatures 


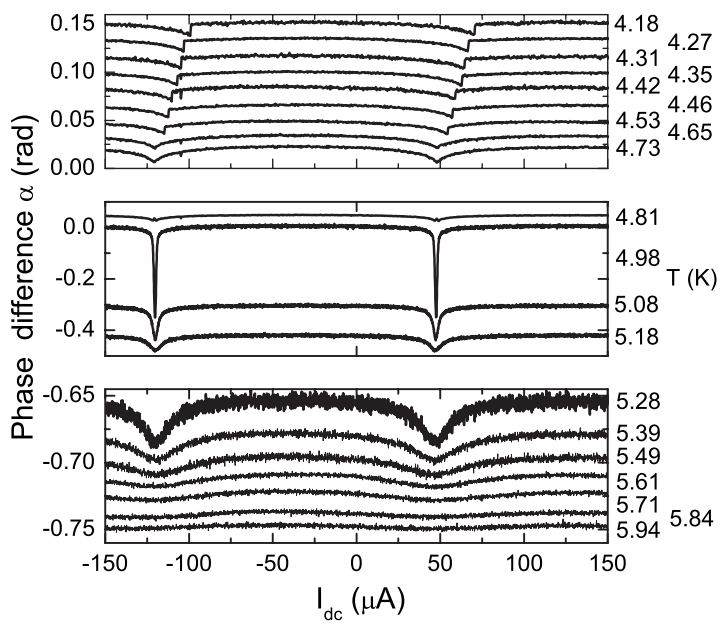

FIG. 3. Measured phase difference $\alpha$ as a function of $I_{d c}$ through the tank circuit coil for different temperatures for a nanobridge with a width of $60 \mathrm{~nm}$. A transition from discontinuous to continuous $\alpha^{\prime}\left(I_{d c}\right)$ characteristics can be noted for increasing temperatures.

to sawtoothlike dependencies at lower temperatures can be discerned. This is in agreement with predictions made by Kupriyanov and Lukuchev, ${ }^{20}$ as was described in the Introduction. From the maximum value of $\beta f(\phi)$, the critical current of the nanobridge can be estimated [Eq. (1)]. Close to the crossover from the single- to multivalued CPR regimes $(T=4.98 \mathrm{~K})$, this yields $I_{c} \approx 12 \mu \mathrm{A}$.

Similar measurements to the ones reported above for the $60 \mathrm{~nm}$ wide $\mathrm{Nb}$ nanobridge were performed for a bridge with a width of $30 \mathrm{~nm}$ and a length of $150 \mathrm{~nm}$, incorporated in a coil with $L_{L} \approx 5.4 \mathrm{pH}$. At $T=4.2 \mathrm{~K}$, single-valued CPRs were determined for this sample. For decreasing temperatures, the transition to phase slippage in the structure was observed around $T=2.65 \mathrm{~K}$. Based on the maximum value of $\beta f(\phi)$, it was determined that, close to the crossover, $I_{c} \approx 12 \mu \mathrm{A}$, which, within the accuracy of the method, is the same as for the $60 \mathrm{~nm}$ wide structure.

As described in the Introduction, it is expected that for narrow nanobridges, the nature of the CPR at fixed tempera-

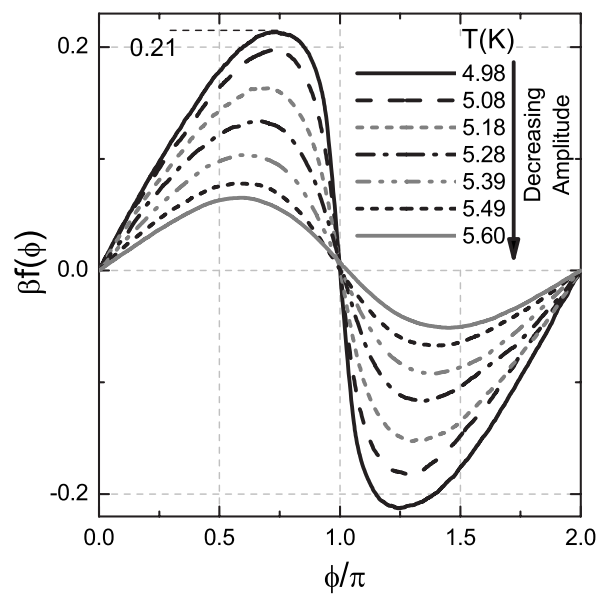

FIG. 4. Calculated CPRs in the single-valued regime for the $\alpha\left(I_{d c}\right)$ characteristics shown in Fig. 3.

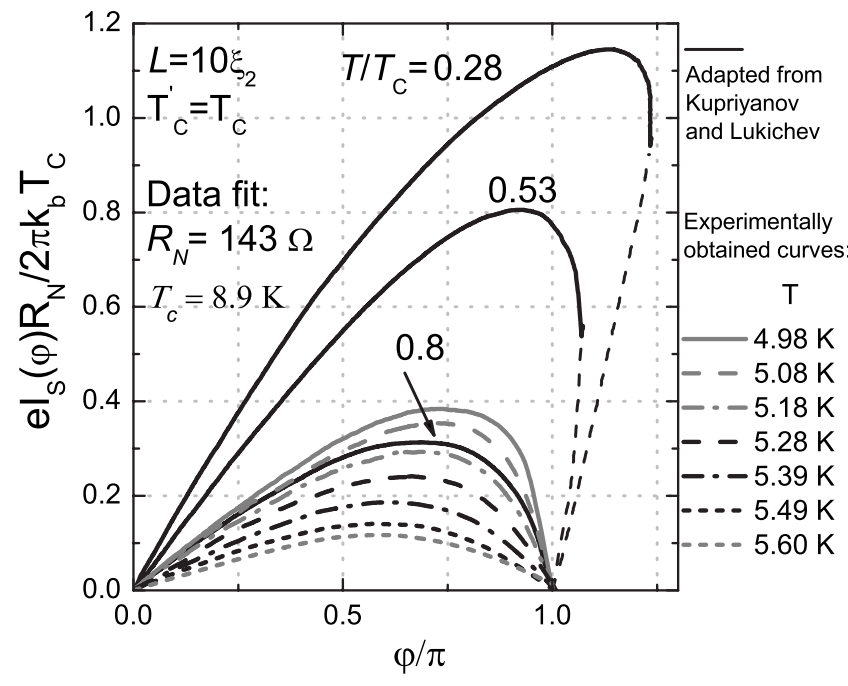

FIG. 5. Experimentally determined CPRs, as shown in Fig. 4, in units of $e I_{s}(\phi) R_{N} / 2 \pi k_{b} T_{c}$ and calculated CPRs for a bridge with $L=10 \xi$ and $T_{c}^{\prime}=T_{c}$. The data were fitted with the following parameters: $R_{N}=143 \Omega$ and $T_{c}=8.9 \mathrm{~K}$.

tures is dependent on the length of the structures. The bridges described above, however, have identical geometrical lengths. The difference in the nature of the CPR at $T=4.2 \mathrm{~K}$ can be explained by the fact that the bridges have a hyperbolic shape. For such structures, the effective length $\left(L_{e f f}\right)$, which can be significantly larger than the geometrical length, is dependent on the width of the bridge. ${ }^{3}$ The relative effective length of the nanobridges, determined by the relation of $L_{\text {eff }}$ to $\xi(T)$, can thus differ significantly from one structure to the other. The fact that for both samples the critical currents close to the transition from multi- to singlevalued CPRs were determined to be approximately identical could be explained by the fact that $I_{c}$ is merely a function of the effective dimensions of the structure, ${ }^{3}$ i.e., for both structures, the crossover is expected for similar values of $L_{\text {eff }} / \xi(T)$.

In both cases, $I_{c}$ was determined as the maximum current at the point of transition from single- to multivalued CPRs. If the walls of the bridge are ideal, it can be expected that superconductivity in the structure is destroyed by supercurrent induced depairing effects. To test this suggestion, we plotted the experimental data in units of $e I_{s}(\phi) R_{N} / 2 \pi k_{b} T_{c}$, together with the theoretical curves calculated in Ref. 20. The results of this comparison are shown in Fig. 5.

From this figure, it can be concluded that there is good qualitative agreement in the shapes of the curves. To obtain quantitative agreement, a value of $R_{N}$ of more than an order of magnitude larger than typically measured values was used. A suggested explanation for this is that the destruction of superconductivity in the structure occurs due to the penetration of Abrikosov vortices into the bridge rather than by depairing by a bias current. This mechanism of destruction is achieved at smaller supercurrent densities and, like in ordinary superconducting films, is not dependent on the length of the structure but on the probability of vortex nucleation in an inhomogeneity in the sidewalls of the bridge. This could thus 
be another explanation for the similarity of the critical currents of the bridges near the crossover.

Based on theory, phase slippage in the structure results in both intrinsic ${ }^{27}$ and thermal hysteresis ${ }^{28,29}$ in the $I V$ characteristics of a superconducting nanobridge. In practice, this means that, in order to obtain electronic components with nonhysteretic $I V$ characteristics, the nature of the CPR should be single valued. In the proceeding part, the results obtained from the CPR measurements will be qualitatively compared to the electronic properties of realized SQUIDs based on similar nanobridges.

For single-valued CPRs, the electronic properties of the $\mathrm{Nb}$ nanobridges are expected to resemble conventional Josephson junctions. In this case, hysteretic $I V$ characteristics are expected for $\beta_{c}=2 \pi R^{2} C I_{c} / \Phi_{0}$, with $R$ and $C$ the bridge resistance and capacitance, respectively. At $T=4.2 \mathrm{~K}$, typical values for $R$ are 10-20 $\Omega$. The capacitance of a nanobridge is approximated by a parallel plate configuration consisting of the two banks of the electrodes: $C=\epsilon_{0} d / A$, with $\epsilon_{0} \approx 8.8 \times 10^{-12} \mathrm{~F} / \mathrm{m}$ the permittivity of free space, $d$ the separation corresponding to the geometrical bridge length $(\approx 150 \mathrm{~nm})$, and $A$ the transverse area of the banks $(\approx 5 \mu \mathrm{m} \times 50 \mathrm{~nm})$. This yields a negligibly small value of $C \approx 10^{-17} \mathrm{~F}$. Given these values for $R$ and $C, \beta_{c} \ll 1$ for $I_{c}=12 \mu \mathrm{A}$. Based on this discussion, patterned $\mathrm{Nb}$ nanobridges with $I_{c}=12 \mu \mathrm{A}$ at $T=4.2 \mathrm{~K}$ are likely to exhibit nonhysteretic $I V$ characteristics at this temperature.

In Fig. 6, the $I V$ characteristics at $T=4.2 \mathrm{~K}$ of two SQUIDs based on $\mathrm{Nb}$ nanobridges of different widths (A: $w \approx 65 \mathrm{~nm}, \mathrm{~B}: w \approx 30 \mathrm{~nm})$ but similar lengths $(\approx 150 \mathrm{~nm})$ and heights $(\approx 50 \mathrm{~nm})$ are displayed. Significant hysteresis can be noted in the curve belonging to device $\mathrm{A}$, for which $I_{c} \approx 40 \mu \mathrm{A}$ was determined. The characteristic of SQUID B, with $I_{c} \approx 20 \mu \mathrm{A}$, is nonhysteretic. Similar results have been obtained at $T=4.2 \mathrm{~K}$ for SQUIDs based on bridges with different critical currents. ${ }^{11}$ Typically, at this temperature, the $I V$ characteristics of devices with $I_{c} \lesssim 25 \mu \mathrm{A}(\approx 2 \times 12 \mu \mathrm{A})$ are nonhysteretic. For SQUIDs with larger critical currents, the characteristics are hysteretic. Since $\beta_{c} \ll 1$ for all these devices, it can be concluded that the onset of this hysteresis is determined by the transition from single- to multivalued CPRs in the nanobridges and not by the resistive and capacitive shunts. dc SQUIDs are commonly operated by shunting the device at a current $I>I_{c}$ and measuring the fielddependent voltage. Since this type of operation requires nonhysteretic $I V$ characteristics, based on the described results, a

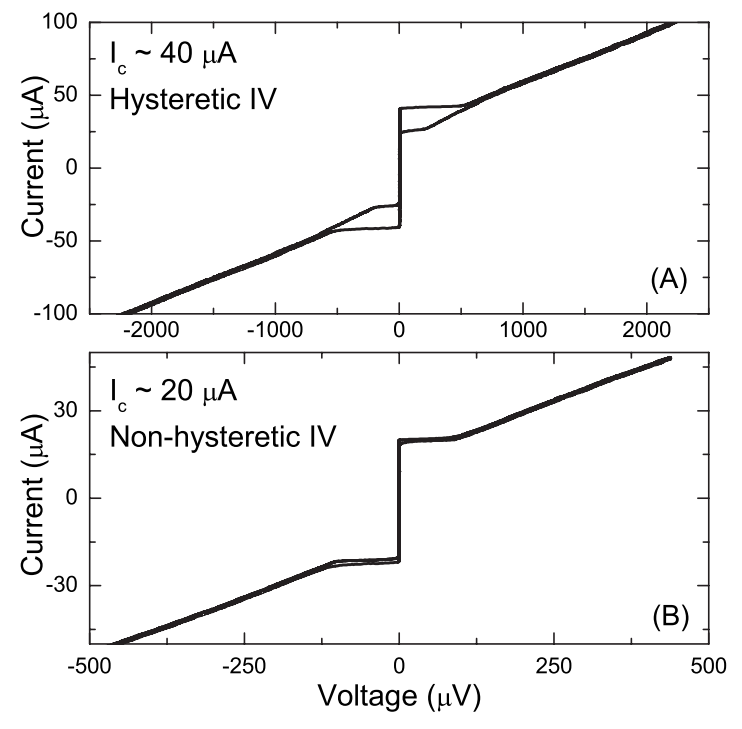

FIG. 6. $I V$ characteristics at $T=4.2 \mathrm{~K}$ of two different $\mathrm{Nb}$ nanobridge based SQUIDs. The hysteretic characteristic shown in part (A) belongs to a SQUID with $I_{c} \approx 40 \mu \mathrm{A}$ and the nonhysteretic one (B) to a device with $I_{c} \approx 20 \mu \mathrm{A}$.

practical limit can be set to the critical current of applicable $\mathrm{Nb}$ nanobridge based SQUIDs $\left(I_{c} \lesssim 25 \mu \mathrm{A}\right)$.

In conclusion, we have presented direct measurements of the temperature dependent deformation (from sinusoidal to sawtoothlike) of the current-phase relationship in niobium nanobridges. At low temperatures, single-valued characteristics reminiscent of Josephson-like behavior are measured. A transition to multivalued characteristics, which are expected in the case of phase slippage in the structure, was observed at $T=4.89 \mathrm{~K}$ for a $60 \mathrm{~nm}$ wide bridge and at $T=2.65 \mathrm{~K}$ for a $30 \mathrm{~nm}$ wide bridge. Finally, given the presented currentvoltage characteristics of SQUIDs based on similar nanobridges, it can be concluded that the possible hysteresis in these characteristics is related to phase slippage in the structure. For SQUIDs based on nanobridges with single-valued CPRs, nonhysteretic $I V$ characteristics are observed.

\section{ACKNOWLEDGMENTS}

This work was supported by the Netherlands Organization for Scientific Research (NWO) through a VIDI grant, the ESF Pishift program, and the STW NanoNed program.
${ }^{1}$ B. D. Josephson, Phys. Lett. 1, 251 (1962).

${ }^{2}$ W. Anderson and J. Rowell, Phys. Rev. Lett. 10, 230 (1963).

${ }^{3}$ K. K. Likharev, Rev. Mod. Phys. 51, 101 (1979).

${ }^{4}$ A. A. Golubov, M. Y. Kupriyanov, and E. Il'Ichev, Rev. Mod. Phys. 76, 411 (2004).

${ }^{5}$ M. Jamet, W. Wernsdorfer, C. Thirion, D. Mailly, V. Dupuis, P. Melinon, and A. Perez, Phys. Rev. Lett. 86, 4676 (2001).

${ }^{6}$ J. Gallop, P. W. Joseph-Franks, J. Davies, L. Hao, and J. MacFar- lane, Physica C 368, 109 (2002).

${ }^{7}$ S. K. H. Lam and D. Tilbrook, Appl. Phys. Lett. 82, 1078 (2003).

${ }^{8}$ J. Dechert, K. Krischker, T. Goddenhenrich, M. Muck, and C.

Heiden, IEEE Trans. Appl. Supercond. 7, 1051 (1997).

${ }^{9}$ C. Veauvy, K. Hasselbach, and D. Mailly, Rev. Sci. Instrum. 73, 3825 (2002).

${ }^{10}$ K. Chen, P. Magnelind, P. Larsson, A. Y. Tzalenchuk, and Z. G. Ivanov, Physica C 372-376, 63 (2002). 
${ }^{11}$ A. G. P. Troeman, H. Derking, B. Borger, J. Pleikies, D. Veldhuis, and H. Hilgenkamp, Nano Lett. 7, 2152 (2007).

${ }^{12}$ G. N. Gol'tsman, O. Okunev, G. Chulkova, A. Lipatov, A. Semenov, K. Smirnov, B. Voronov, A. Dzardanov, C. Williams, and R. Sobolewskib, Appl. Phys. Lett. 79, 705 (2001).

${ }^{13}$ D. E. Prober, Appl. Phys. Lett. 62, 17 (1993).

${ }^{14}$ J. E. Mooij and C. J. P. M. Hermans, J. Therm. Stresses 7, 219 (2005).

${ }^{15}$ J. E. Mooij and Y. V. Nazarov, Nat. Phys. 2, 169 (2006).

${ }^{16}$ I. O. Kulik and A. N. Omelyanchuk, Zh. Eksp. Teor. Fiz. Pis'ma Red. 21, 216 (1975).

${ }^{17}$ M. C. Koops and G. V. van Duyneveldt, and R. de Bruyn Ouboter, Phys. Rev. Lett. 77, 2542 (1996).

${ }^{18}$ M. L. Della Rocca, M. Chauvin, B. Huard, H. Pothier, D. Esteve, and C. Urbina, Phys. Rev. Lett. 99, 127005 (2007).

${ }^{19}$ K. K. Likharev and L. A. Yakobson, Pis'ma Zh. Tekh. Fiz. 45, 1503 (1975).

${ }^{20}$ M. Y. Kupriyanov and V. F. Lukuchev, Fiz. Nizk. Temp. 7, 281 (1981).
${ }^{21}$ M. Y. Kupriyanov, K. K. Likharev, and L. A. Maslova, Proceedings of the 14th International Conference on Low Temperature Physics, 1975 (unpublished), p. 104.

${ }^{22}$ V. I. Gubankov, V. P. Koshelets, and G. A. Ovsyannikov, Zh. Eksp. Teor. Fiz. 71, 348 (1976).

${ }^{23}$ S. S. Pei, J. E. Lukens, and R. D. Lukens, Appl. Phys. Lett. 36, 88 (1980).

${ }^{24}$ A. H. Silver and J. E. Zimmerman, Phys. Rev. 157, 317 (1967).

${ }^{25}$ R. Rifkin and B. S. Deaver, Phys. Rev. B 13, 3894 (1976).

${ }^{26}$ E. Il'ichev, V. Zakosarenko, L. Fritzsch, H. E. Hoenig, H. G. Meyer, A. B. Zorin, V. V. Khanin, M. GÄotz, A. B. Pavolotsky, and J. Niemeyer, Rev. Sci. Instrum. 72, 1882 (2001).

${ }^{27}$ A. A. Kadin, L. N. Smith, and W. J. Skocpol, J. Low Temp. Phys. 38, 497 (1980).

${ }^{28}$ W. J. Skocpol, M. R. Beasley, and M. Tinkham, J. Appl. Phys. 45, 4045 (1974).

${ }^{29}$ A. M. Kadin, W. J. Skocpol, and M. Tinkham, J. Low Temp. Phys. 33, 481 (1978) 